\title{
Object Oriented Prompted Play (O2P2): A Pragmatic Approach to Interactive Narrative
}

\author{
Daniel Roberts and Mark Wright \\ EdVEC (Edinburgh Virtual Environment Centre) \\ The University of Edinburgh, Edinburgh EH9 3JZ, UK \\ \{Daniel.Roberts, Mark.Wright\}@ed.ac.uk \\ www.edvec.ed.ac.uk
}

\begin{abstract}
We describe an interactive narrative system that embodies Object Oriented Prompted Play (O2P2). This means that behaviours, attributes, and crucially stories are attached to objects in the story world. The approach is inspired by children's social play where narrative emerges from collaborative, improvised negotiation. The object oriented architecture is appropriate for improvisation, imposing little predefined structure. Our goal is not the modelling, understanding and generation of narrative but the computer aided support for the improvisation process from which narrative emerges. Although based on play and children's material, we believe the system is applicable more widely. Ours is a pragmatic approach that strikes a balance between the culturally fundamental activities of "play" and "story telling".
\end{abstract}

\section{Introduction}

In this paper we describe a system that represents a new approach to interactive narrative: Object Oriented Prompted Play (O2P2). This paradigm occupies the middle ground between open-ended play and structured narrative. Our goal is not to create a system which encodes, models, understands or generates a definitive narrative, but to create a system that facilitates collaborative play from which improvised narrative emerges. The narrative is object oriented in the sense that behaviours, attributes, and most importantly, stories are attached to objects in the scene. An object oriented architecture is appropriate for improvisation because its distributed nature does not impose much pre-defined structure. In principle, any type of story could be attached to an object, but we envisage them as being low-level subplots involving the object and one or more characters. For example, a seesaw may have a story attached that involves two characters playing together, or using the seesaw as a lever. The user is able to introduce new objects to the scene, with subplots attached that are integrated in to the template plot. The user controls the characters in the story, and, through them, can interact with the objects that are present. Prompted play is inspired by the research and observations of children's 'collaborative play'[7]. Such play is a socially embedded stream of interactions [2]. Narrative emerges during such play as the result of an unpredictable, improvised and constantly renegotiated process. 
There is no master script, no child or other agent is totally in control of this process. The embedded, distributed and unpredictable nature of the interactions and the narrative which emerges means that the application of traditional global structural reductionist approaches of deconstruction, analysis and modelling are problematic. We therefore focus not on narrative modelling, understanding and generation but on computer aided support for the improvisation process.

Another insight we use from research on play, is that global coherence of these emergent narratives is aided by scaffolding, where gross context is provided, and that local sequential coherence is aided by metaplay or dialogic strategies, where children narrate in or out of character to facilitate negotiation of the direction of the narrative.

Our system aims to aid the improvisation process by embodying the principles of scaffolding, metaplay and dialogic processes. The system as a whole is a kind of 'play facilitator' - a good analogy is that of a kindergarten assistant who suggests an initial context and scenario (scaffolding), moves things on when a scenario finishes, and ensures that the narrative doesn't flag, either by taking over and telling the story for a while, or by prompting the children (or user) with new ideas. The embedding of stories and behaviours in objects facilitates improvisation as well as allowing the system to engage in metaplay/dialogic contributions to affect the flow of the narrative.

\section{Related Work}

State of the art approaches to interactive narrative aim to solve perennial problems of the field - high level issues of linguistic comprehension, narrative coherence, and dramatic tension. These are constant themes, running from Propp's work on modelling the grammar of narrative [5], through to present day systems such as Mateas and Stern's Facade [3] and Szilas' IDTension [8]. Both of these are sophisticated systems that aim to provide a structured narrative experience analogous to that of a good novel or movie - one that follows the traditional 'dramatic arc'. Parallels can be drawn between our system and theirs: in our system, a conflict must be resolved before another can start, and the actions of the user dictate which conflict/resolution pair occurs next. Facade functions in a similar way - input from the user affects the actions of the other characters in the interactive drama, and determines which 'beat' happens next. IDTension has as a component a 'narrative logic' that presents to the user all possible actions that are available to him. This is similar to our concept of a narrator who suggests actions to the user at appropriate junctures in the plot.

However, despite these similarities, our approaches are fundamentally different. We are not aiming for a narrative experience that approximates that of a film or a book. Rather, we hope to create an experience that is closer to the narrative that emerges from play. By aiming for a different, less stringent kind of narrative, namely the kind that emerges from collaborative play, we hope to avoid the strictures of more conventional narrative modalities. There is no 'master plot' in our system; simply a 'template plot' that, in conjunction with 


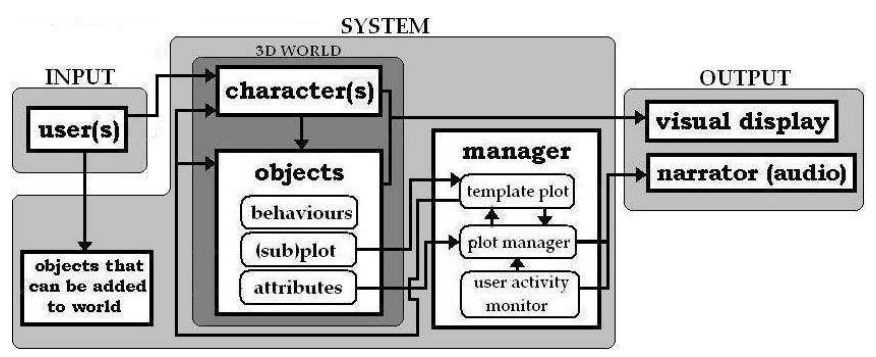

Fig. 1. Architecture of the system

prompts from the narrator and subplots added by the user, provides a loose narrative context within which the user can play.

Systems which also aim to involve play in the interactive narrative experience include StoryMat [6], a playmat that records the verbally improvised stories of children, and plays back those recorded by others at appropriate junctures. StoryMat shares our desire to mediate improvisational play, but comes at the problem from a different angle. The KidsRoom [1] was an interactive, narrative playspace for children. The actions of the children, who were in a real bedroom overlooked by several video cameras, were interpreted by computer vision tracking and action recognition algorithms. Recognition of the movements initiated a structured narrative experience, in which the scope for improvisation was limited. In Computer-Animated Improvisational Theater [4], the improvisation is performed by the system rather than the user. The user gives directions to the animated characters - these directions can be anywhere between very specific ("hop over there energetically") and very general ("move over there in whatever way you want"). A general instruction means that, in the case of the examples given, the character must improvise the method of locomotion.

\section{Sketch Of The System}

Figure 1 is a schematic representation of our interactive narrative system. Below is an explanation of how the system will work. Figure 2 gives an example of a user's experience of the system, using a plot based on a Tiny Planets episode.

User Agency - The user's agency in the system consists of two components: firstly, the ability to control the character or characters that are present in the world; secondly, the ability to add new objects (with subplots attached) to the world. The addition of new objects to the world takes place as a drag-anddrop operation from a menu of objects appropriate to the current world state. The user does not design the plots attached to objects, or indeed the objects themselves. In our system the plots, objects and characters are taken from the TV series Tiny Planets. A conventional author/3D modeller who was familiar with our system could just as easily design them.

The 3D Virtual World - All action that takes place in a plot is represented in a $3 \mathrm{D}$ virtual world, consisting of the characters and objects involved. The 


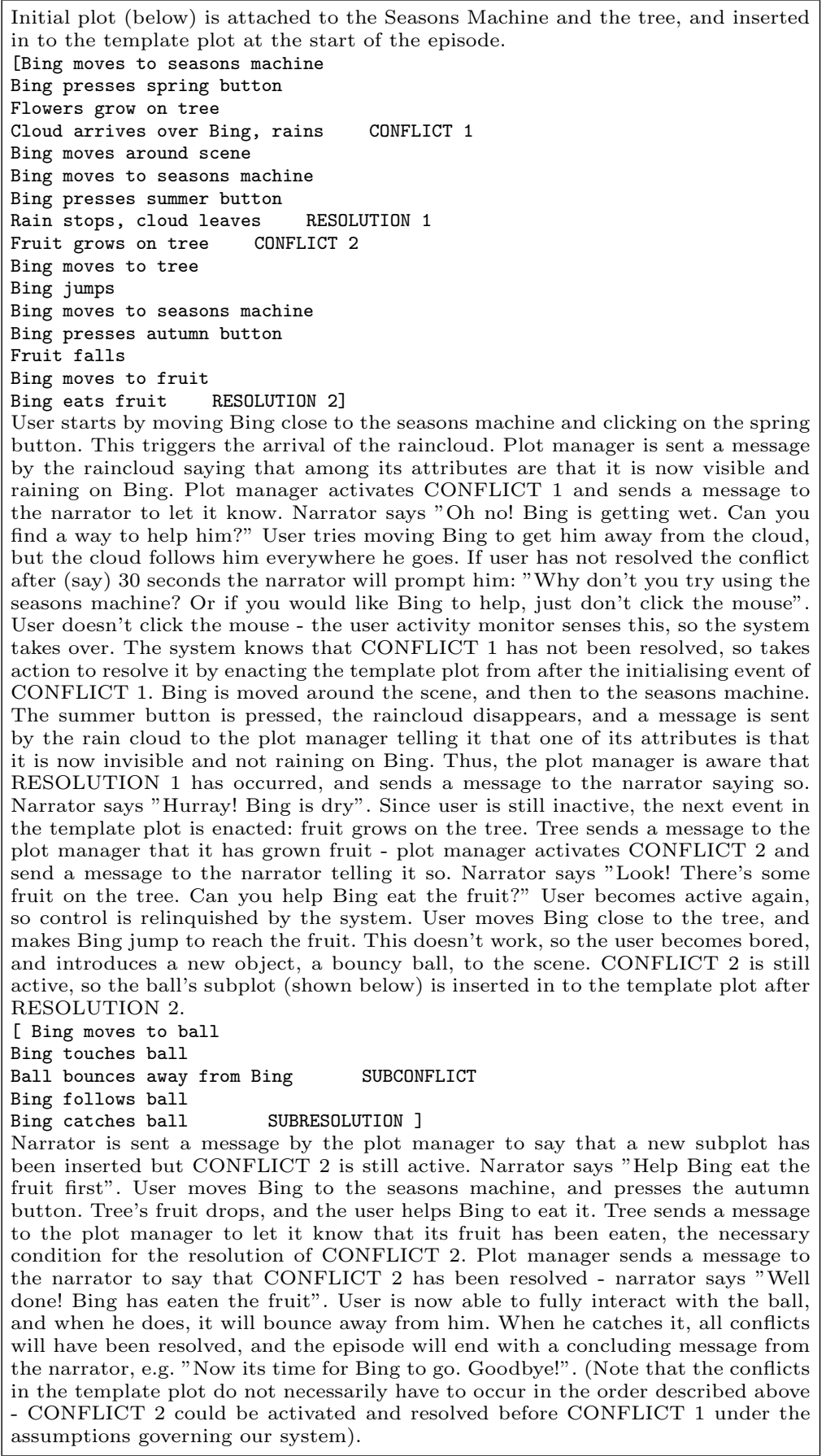

Fig. 2. Walkthrough of user experience 
objects in the world have behaviours, attributes, and plots attached. Behaviours, as the name suggests, dictate the behaviour of an object in certain situations. These behaviours alter the attributes of the object. The plot attached to an object become part of the template plot when the object is introduced to the world.

Representation Of Plot - A plot is modelled as a linear list of events. These events in the list correspond to animations that are played in the $3 \mathrm{D}$ virtual world. Some events are triggers for behaviours (trigger events), and need to be initiated by the plot manager. The rest (behaviour events) occur as a result of these triggers, and are part of a behaviour, meaning that they do not need to be initiated by the plot manager. As already mentioned, plots are attached to objects in the scene. The template plot is initialised as the list of events that is attached to objects present in the world at the commencement of the story. Within the list of events that constitutes a plot, certain events are identified as the initiation of a conflict, and others as the resolution of a conflict.

Management Of Plot - To make the management of the plot more tractable, two simplifying assumptions have been made: firstly, that no more than one conflict can be 'active' (i.e. unresolved) at any one time; secondly, that the order in which conflict/resolution pairs occur is unimportant. These assumptions may seem naive, but they are not unreasonable in the context of the simple, problemsolving narratives of the Tiny Planets series. Once our system has been validated using these initial simplifying assumptions, they will be discarded in favour of a more realistic approach.

The plot manager takes care of the interplay between user, system and template plot. When the user is active, the plot manager keeps track of any conflicts or resolutions from the template plot that the user may trigger, and keeps him updated on the situation via the narrator. When the user is inactive, the plot manager steps through the template plot until he becomes active again. The functioning of the plot manager is made clearer by the example in Figure 2.

Introduction Of New Objects/Subplots - A subplot attached to a newly introduced object is represented (like the template plot) as a list of events. This list is inserted in to the template plot list either immediately after the current event (if no conflict is active), or after the event that resolves the current conflict (if a conflict is active).

The Narrator - The narrator gives verbal prompts to the user, as well as a more brief text prompt at the bottom of the screen. It can be viewed as the 'voice' of the kindergarten assistant described in the introduction - it mediates the processes of scaffolding and metaplay.

\section{Conclusions And Future Work}

In this paper, we have described a new approach to interactive narrative, Object Oriented Prompted Play (O2P2). We have also given a sketch of an interactive narrative system that implements this concept. Our pragmatic approach avoids difficult issues of narrative coherence and understanding by fashioning an ex- 
perience in which play is the primary constituent. The stories attached to the objects in the 3D virtual world, along with prompts from the narrator, apply a narrative structure to this play, resulting in a system that combines the desirable attributes of open-ended play with those of structured narrative. We are applying this system to short, simple narratives of a problem solving nature, but we see it as having applications in many different situations.

At present, we have implemented an interactive pilot of the 3D Tiny Planets world, with behaviours embedded in objects and narrator functionality. Work for the immediate future includes following this implementation through to completion, and testing the system on users. More distant goals are to investigate how the system would work if we removed the simplifying assumptions that were made, to explore character intelligence and growth throughout the narrative, and to deal with the issue of the management of the system's growth as new objects are added by the user.

Finally, we believe that the key to providing more engaging experiences from interactive media in the future is to strike a balance between the culturally fundamental activities of "story telling" and "play". Object Oriented Prompted Play (O2P2) is a paradigm which offers such a balance.

\section{Acknowledgements}

TINY-IN is funded by EPSRC grant GR/R54361/01 under the DTI Broadcast Link initiative. Collaborators are KMi (Open University), R. Joiner (University of Bath) and Pepper's Ghost Productions. Thanks to Carl Goodman (PGP), Peter Stansfield (Wavecrest Systems Ltd.) and Criterion Software Ltd for the use of RenderWare ${ }^{T M}$.

\section{References}

1. Bobick, A.F., Intille, S.S., Davis, J.W., Baird, F., Pinhanez, C.S., Campbell, L.W., Ivanov, Y.A., Schutte, A. and Wilson, A.: Perceptual User Interfaces: the KidsRoom. In Communications of the ACM 43(3) (March 2000) 60-61

2. Dourish, P.: Where the Action Is: The Foundations of Embodied Interaction. Massachusetts Institute of Technology (2001)

3. Mateas, M. and Stern, A.: Architecture, Authorial Idioms and Early Observation of the Interactive Drama Facade. Tech Report CMU-CS-02-198, School of Computer Science, Carnegie Mellon University, Pittsburgh, PA (December 2002)

4. Hayes-Roth, B., Brownston, L., and van Gent, R.: Multiagent collaboration in directed improvisation. Stanford University Report KSL-94-69 (1994)

5. Propp, V.: Morphology of the Folktale. In International Journal of American Linguistics, Vol. 24, Nr. 4, Part III, Bloomington, IN, (1958)

6. Ryokai, K. and Cassell, J.: Computer Support for Children's Collaborative Fantasy Play and Storytelling. In Proceedings of CSCL '99 (1999)

7. Sawyer, R.K.: Improvisation and Narrative. In Narrative Inquiry, 12(2), John Benjamins B.V. Amsterdam (2002) 319-349

8. Szilas, N.: IDtension: a narrative engine for Interactive Drama. In Proc. of TIDSE 2003 (Darmstadt, Germany), AAAI Press, 72-76(March 2003) 72-76 